Concept Paper

\title{
Electromagnetic Wave Scattering by Small Impedance Particles of an Arbitrary Shape and Applications
}

\author{
Alexander G. Ramm \\ Mathematics Department, Kansas State University, Manhattan, KS 66506, USA; \\ E-Mail: ramm@math.ksu.edu
}

Received: 22 September 2013 / Accepted: 16 January 2014 / Published: 7 February 2014

\begin{abstract}
The proposal deals with electromagnetic (EM) wave scattering by one and many small impedance particles of an arbitrary shape. Analytic formula is derived for EM wave scattering by one small impedance particle of an arbitrary shape and an integral equation for the effective field in the medium where many such particles are embedded. These results are applied for creating a medium with a desired refraction coefficient. The proposed theory has no analogs in the literature. (Mathematical Subject Classiffication: 35J05, 35J25, 65N12, 78A25, 78A48.)
\end{abstract}

Keywords: Electromagnetic wave scattering; small impedance particles; many-body scattering problem; refraction coefficient; permeability; creating materials with a desired refraction coefficient

\section{Statement of the Problem}

Let

$$
\begin{gathered}
\nabla \times E=i \omega \mu H, \quad \nabla \times H=-i \omega \epsilon E \\
E=E_{0}+v_{E}, \quad E_{0}=\xi e^{i k \alpha \cdot x}, \quad \alpha \cdot \xi=0 \\
\frac{\partial v_{E}}{\partial r}-i k v_{E}=o\left(\frac{1}{r}\right), \quad r=|x| \rightarrow \infty
\end{gathered}
$$

Equation (1) hold in $D^{\prime}:=\mathbb{R}^{3} \backslash D, D$ is a bounded domain, $a:=\frac{1}{2} \operatorname{diam} D, k a \ll 1$, i.e., $D$ is a small body, the boundary $S$ of $D$ is $C^{1, \gamma}$-smooth, i.e., in the local coordinates the equation of $S$ is $z=f(x, y)$ and $f$ has first derivative, satisfying the Hölder condition with exponent $0<\gamma \leq 1$. The impedance boundary condition on $S$ is

$$
[N,[E, N]]=\zeta[N, H]
$$


where $N$ is the unit normal to $S$ pointing out of $D, \zeta$ is the impedance, $\operatorname{Im} \zeta \geq 0, \zeta=$ const. Vector $\xi$ in Equation (2) is constant, $\alpha \in S^{2}, S^{2}$ is the unit sphere in $\mathbb{R}^{3}$, condition $\alpha \cdot \xi=0$ implies $\nabla \cdot E_{0}=0$. The $k=\omega \sqrt{\epsilon \mu}$ is the wave number, $\omega$ is the frequency, $\epsilon$ and $\mu$ are dielectric and magnetic constants.

The problem PI (Principal Investigator) proposes to investigate is the electromagnetic (EM) wave scattering problem by one and many small impedance bodies (particles) of an arbitrary shape.

If there are $M$ small particles, then $D:=\cup_{m=1}^{M} D_{m}, D^{\prime}:=\mathbb{R}^{3} \backslash D, \zeta=\frac{\zeta_{m}}{a^{\kappa}}$ on $S_{m}$, the surface of $D_{m}, \zeta_{m}:=h\left(x_{m}\right), x_{m} \in D_{m}$ is an arbitrary point in $D_{m}, h(x), \operatorname{Im} h \geq 0$ is a given continuous function in $\mathbb{R}^{3}$, and $\kappa \in[0,1)$ is a parameter. The function $h(x)$ and the parameter $\kappa$ can be chosen by the experimentalist as he wishes.

Equations (1)-(4) can be reduced to finding vector $E$, and if $E$ is found then $H$ is found from the equation $H=\frac{\nabla \times E}{i \omega \mu}$. The problem for $E$ is:

$$
\nabla \times \nabla \times E=k^{2} E, \quad k^{2}:=\omega^{2} \epsilon \mu
$$

where we have assumed $\nabla \times \mu H=\mu \nabla \times H$

$$
[N,[E, N]]=\frac{\zeta}{i \omega \mu}[N, \nabla \times E] \text { on } S
$$

and Equations (2) and (3) remain valid.

The first basic result PI proposes to obtain is an analytic, closed-form formula for the EM field scattered by the small impedance body $D$ of an arbitrary shape.

The second basic result PI proposes to obtain is a numerical method for solving many-body EM wave scattering problem in the case of $M$ small impedance bodies of an arbitrary shape, where $M$ ranges from $O(10)$ to $O\left(10^{12}\right)$. The usual approach, based on solving boundary integral equations, is not feasible when $M$ is large. A new approach and new ideas are needed. These are described in Section 2.

The third basic result PI proposes to derive is an integral equation for the effective field in the medium in which many small impedance bodies (particles) are embedded. This allows PI to derive an analytic formula for the refraction coefficient of the above medium. PI proposes to give a method for creating materials with a desired refraction coefficient by embedding many small impedance particles in the given medium. The distribution of the small particles and their boundary impedances determine the desired refraction coefficient.

PI and his Ph.D students (research assistants) plan to obtain numerical implementation of the PI's theory, developed in this proposal. Since the number of small particles is very large, the numerical implementation requires solving very large linear algebraic systems. PI plans to use GMRES method ([18]) for solving such systems and PETSC libraries to do the computations in parallel ([1]).

\section{Description of PI's Approach}

Let us look for the solution Equations (1)-(4) of the form

$$
E(x)=E_{0}(x)+\nabla \times \int_{S} g(x, t) \sigma(t) d t
$$

where $\sigma(t)$ is some tangential to $S$ field and $d t$ is the element of the surface area. Let

$$
Q:=\int_{S} \sigma(t) d t
$$


Then Equation (7) can be written as

$$
E(x)=E_{0}(x)+\left[\nabla g\left(x, x_{1}\right), Q\right]+\nabla \times \int_{S}\left(g(x, t)-g\left(x, x_{1}\right)\right) \sigma(t) d t
$$

where $x_{1} \in D$ is an arbitrary point and one has used the formula $\nabla \times g\left(x, x_{1}\right) Q=[\nabla g, Q]$, where $[A, B]=A \times B$ is the vector product, $A \cdot B=(A, B)$ is the scalar product. PI's main idea is to prove that if $k a \ll 1$ (small $D$ ) and $d:=\left|x-x_{1}\right| \gg a$, then

$$
\left|\nabla \times \int_{S}\left(g(x, t)-g\left(x, x_{1}\right)\right) \sigma(t) d t\right| \ll\left|\left[\nabla g\left(x, x_{1}\right), Q\right]\right|
$$

Therefore, the problem of finding a function $\sigma(t)$ from the boundary condition Equation (6), which amounts to solving a boundary integral equation (bie), the problem Equations (2), (3), (5), (6) is reduced to finding one pseudovector $Q$. Note that since $E$ is a vector $\sigma$ has to be a pseudo-vector field. PI derives an asymptotic analytic formula, as $a \rightarrow 0$, namely

$$
Q=-\frac{\zeta|S|}{i \omega \mu} \tau \nabla \times E_{0}\left(x_{1}\right), \quad a \rightarrow 0
$$

where $|S|$ is the surface area of $S$ and

$$
\tau:=\left(\tau_{j p}\right):=\delta_{j p}-\frac{1}{|S|} \int_{S} N_{j}(s) N_{p}(s) d s
$$

$N_{j}(s)$ is the $j$-th component of the unit normal $N(s)$ at the point $s \in S$. Thus the solution to the EM wave scattering problem Equations (2), (3), (5), (6) is

$$
E(x)=E_{0}(x)-\frac{\zeta|S|}{i \omega \mu}\left[\nabla_{x} g\left(x, x_{1}\right), \quad \tau \nabla \times E\left(x_{1}\right)\right]
$$

This formula is valid if $k a \ll 1$ and $\left|x-x_{1}\right| \gg a$. To derive formula Equation (13), PI plans to use boundary condition Equation (6) not for solving the result from Equation (6) equation for $\sigma$, but for finding asymptotic formula Equation (11).

If there are $M \gg 1$ small bodies, then PI looks for the solution to problem Equations (2), (3), (5), (6) with $D=\cup_{m=1}^{M} D_{m}$ of the form

$$
E(x)=E_{0}(x)+\sum_{m=1}^{M} \nabla \times \int_{S_{m}} g(x, t) \sigma_{m}(t) d t
$$

PI checks that the right-hand side of Equation (14) satisfies Equations (2), (3), (5) for any $\sigma_{m}$, so that problem Equations (2), (3), (5), (6) is solved if the boundary conditions Equation (6) is satisfied on every $S_{m}, 1 \leq m \leq M$. The field Equation (14) can be written as

$$
E(x)=E_{0}(x)+\sum_{m=1}^{M}\left[\nabla g\left(x, x_{m}\right), Q_{m}\right]+\sum_{m=1}^{M} \nabla \times \int_{S_{m}}\left(g(x, t)-g\left(x, x_{m}\right)\right) \sigma_{m}(t) d t
$$

where $x_{m} \in D_{m}$ are arbitrary points. PI proves that

$$
\left|\nabla \times \int_{S_{m}}\left(g(x, t)-g\left(x, x_{m}\right)\right) \sigma_{m}(t) d t\right| \ll\left[\nabla g\left(x, x_{m}\right), Q_{m}\right]
$$


Thus, the solution to many-body EM wave scattering problem is given by the formula

$$
E(x)=E_{0}(x)-\sum_{m=1}^{M} \frac{\zeta_{m}\left|S_{m}\right|}{i \omega \mu}\left[\nabla_{x} g\left(x, x_{m}\right), \tau_{m} \nabla \times E_{e}\left(x_{m}\right)\right]
$$

where $E_{e}\left(x_{m}\right)$ is the effective field acting on $m$-th particle. Let us assume that small particles $D_{m}$ are of the same shape and size. Then $\tau_{m}=\tau$ and $\left|S_{m}\right|=c_{S} a^{2}$, where $c_{S}>0$ is a constant. For example, the constant $c_{S}=4 \pi$ and $\tau=\frac{2}{3} \delta_{j p}$ if $S_{m}$ are spheres. Let us assume that

$$
\zeta_{m}=\frac{h\left(x_{m}\right)}{a^{\kappa}}
$$

where $h(x)$ is a given continuous function, $\operatorname{Re} h \geq 0$, in a bounded domain $\Omega$ in which the small bodies $D_{m}$ are distributed, and $\kappa \in[0,1)$ is a given parameter. The experimentalist can choose $h(x)$ and $\kappa$ as he wants. Assume also that the number $N(\Delta)$ of small bodies (or points $x_{m}$ ) in any subdomain $\Delta$ is given by the formula

$$
N(\Delta)=\frac{1}{a^{2-\kappa}} \int_{\Delta} N(x) d x(1+o(1)) \quad a \rightarrow 0
$$

where $N(x) \geq 0$ is a continuous in $\Omega$ function that can be chosen by the experimentalist as he wants.

Let us define the effective field acting on $j$-th particle by the formula

$$
E_{e}(x)=E_{0}(x)-\frac{c_{S}}{i \omega \mu} \sum_{m \neq j} a^{2-\kappa} h\left(x_{m}\right)\left[\nabla_{x} g\left(x, x_{m}\right), \tau \nabla \times E_{e}\left(x_{m}\right)\right]
$$

To find vectors $\nabla \times E\left(x_{m}\right)$, PI applies the operator $\nabla \times$ to Equation (20) and then sets $x=x_{j}$ to get:

$$
\nabla \times E\left(x_{j}\right)=\nabla \times E_{0}\left(x_{j}\right)-\frac{c_{S} a^{2-\kappa}}{i \omega \mu} \sum_{m \neq j} h\left(x_{m}\right) \nabla \times\left.\left[\nabla g\left(x, x_{m}\right), \tau \nabla \times E\left(x_{m}\right)\right]\right|_{x=x_{j}}
$$

This is a linear algebraic system for finding vectors $\nabla \times E\left(x_{m}\right) 1 \leq m \leq M$.

If $M$ is very large, say $M \geq 10^{5}$, then one reduces the order of the system Equation (21) as follows. Consider a partition of the domain $\Omega$ in the union of $P \ll M$ small cubes $\Delta_{p}$ of side $b=b(a)$, $1 \leq p \leq P$. Assume that $b \gg d \gg a$, where $d=d(a)$ is the minimal distance between two neighboring particles. Let $x_{p}$ be the center of the cube $\Delta_{p}$, and denote $\nabla \times E\left(x_{p}\right):=A_{p}, \nabla \times E_{0}\left(x_{p}\right)=A_{o p}$. Then Equation (21) can be transformed as follows:

$$
A_{q}=A_{0 q}-\frac{c_{S}}{i \omega \mu} \sum_{p \neq q} \nabla \times\left[\nabla g\left(x, x_{p}\right), \tau A_{p}\right] h\left(x_{p}\right) \sum_{x_{m} \in \Delta_{p}} 1
$$

Using Equation (19) and denoting $\left|\Delta_{p}\right|:=$ volume $\Delta_{p}$, one gets:

$$
a^{2-\kappa} \sum_{x_{m} \in \Delta_{p}} 1=N\left(x_{p}\right)\left|\Delta_{p}\right|, a \rightarrow 0
$$

Thus Equation (22) takes the form

$$
A_{q}=A_{o q}-\frac{c_{S}}{i \omega \mu} \nabla \times \sum_{p \neq q}\left[\nabla g\left(x, x_{p}\right), \tau A_{p}\right] h\left(x_{p}\right) N_{p}\left|\Delta_{p}\right|
$$


As $a \rightarrow 0$, Equation (24) tends to the limiting integral equation

$$
A(x)=A_{0}(x)-\frac{c_{S}}{i \omega \mu} \nabla \times \nabla \times \int_{\Omega} g(x, y) \tau A(y) h(y) N(y) d y
$$

The limiting form of Equation (20) is

$$
E(x)=E_{0}(x)-\frac{c_{S}}{i \omega \mu} \nabla \times \int_{\Omega} g(x, y) \tau \nabla \times E(y) h(y) N(y) d y
$$

To demonstrate possible application of Equation (26) to creating materials with a desired refraction coefficient, apply the operator $\nabla \times \nabla \times$ to Equation (26). The result is

$$
\nabla \times \nabla \times E=k^{2} E_{0}-\nabla \times\left(\operatorname{grad} \operatorname{div}-\nabla^{2}\right) \int_{\Omega} g(x, y) \tau \nabla \times E h(y) N(y) d y
$$

Since $\nabla \times \operatorname{grad}=0$ and $-\nabla^{2} g=\delta(x-y)+k^{2} g$, Equation (27) yields

$$
\nabla \times \nabla \times E=k^{2} E-\frac{c_{S}}{i \omega \mu} \nabla \times(\tau \nabla \times E(x) h(x) N(x))
$$

Assume for simplicity that $h(x)$ and $N(x)$ are constants and $D_{m}$ are spheres, i.e., $\tau=\frac{2}{3} I$. Then Equation (28) yields

$$
\nabla \times \nabla \times E=k^{2} E-\frac{c_{S}}{i \omega \mu} \frac{2}{3} h N \nabla \times \nabla \times E
$$

This means that the refraction coefficient of the new medium which is obtained by embedding of many small impedance balls into $\Omega$, is equal to

$$
n^{2}=1+\frac{c_{S}}{i \omega \mu} \frac{2}{3} h N, \quad n_{0}^{2}=\sqrt{\epsilon \mu}
$$

Since $h$ and $N$ are at our disposal, $\operatorname{Re} h \geq 0$, one can change the original refraction coefficient in a desired direction. For example, suppose one wants to have $\frac{n^{2}}{n_{0}^{2}}=b_{1}+i b_{2}$. Denote $\frac{2}{3} \frac{c_{S} N}{\omega \mu}:=C, h=h_{1}+i h_{2}$. Then Equation (30) yields

$$
b_{1}=\frac{1+C h_{2}}{\left(1+h_{2} C\right)^{2}+h_{1}^{2} C^{2}} ; \quad b_{2}=\frac{h_{1} C}{\left(1+h_{2} C\right)^{2}+h_{1}^{2} C^{2}} ; \quad C:=\frac{2 c_{S} N}{3 \omega \mu}
$$

Formula Equation (28) gives also a recipe for creating materials with a desired magnetic permeability $\mu(x)$. To formulate this recipe apply the operator $\nabla \times$ to the first Equation (1) assuming $\mu=\mu(x)$. This yields

$$
\nabla \times \nabla \times E=k^{2} E+i \omega[\nabla \mu, H]=k^{2} E+\left[\frac{\nabla \mu}{\mu}, \nabla \times E\right], \quad k^{2}=\omega^{2} \epsilon(x) \mu(x)
$$

Compare Equations (28) and (32) assuming that $D_{m}$ are balls of radius $a$, so that $\tau=\frac{2}{3} \delta_{j p}$, and denote $\mu=\mu_{0}$ in Equation (28), while $\mu(x)$ is the magnetic permeability of the new medium in which many small impedance particles are embedded.

Then Equation (28) can be rewritten as

$$
\nabla \times \nabla \times E=\frac{k^{2} E}{1-i \frac{2 c_{S} h(x) N(x)}{3 \omega \mu_{0}}}+\frac{i 2 c_{S}}{3 \omega \mu_{0}} \frac{[\nabla(h(x) N(x)), \nabla \times E]}{1-i \frac{2 c_{S} h(x) N(x)}{3 \omega \mu_{0}}}
$$


Compare Equation (33) with Equation (32) and remember that $H=\frac{\nabla \times E}{i \omega \mu_{0}}$. Then one obtains

$$
\mu(x)=\frac{\mu_{0}}{1-i \frac{2 c_{S} h(x) N(x)}{3 \omega \mu_{0}}} ; \quad \frac{\nabla \mu}{\mu}=\frac{i 2 c_{S} \nabla(h(x) N(x))}{3 \omega \mu_{0}\left(1-i \frac{2 c_{S} h(x) N(x)}{3 \omega \mu_{0}}\right)}
$$

Thus, $\mu(x)$ can be changed by changing $h(x)$ and $N(x)$.

\section{Comparison of the Proposed Research with the Known Results}

EM wave scattering by small bodies is a classical field of theoretical and applied physics and mathematics. Rayleigh in 1871 [17] understood that the main term in the scattered field is given by the dipole radiation. He did not give formulas for the induced dipole moment for a body of an arbitrary shape. This was done about 100 years later by PI ([6-8]). In 1908 Mie [5] gave a solution of the EM wave scattering by one perfectly conducting sphere by separation of variables ([19]). In [2-4], these results are summarized. The origin of these results is the work [17]. There were no results which would give analytic formula, asymptotically exact as $a \rightarrow 0$, for the EM wave field scattered by one small impedance particle (body) of an arbitrary shape. Such a formula PI proposes to derive. There were no methods for solving many-body EM wave scattering problem when the number $M$ of small impedance bodies is very large $M=O\left(10^{5}\right)-O\left(10^{10}\right)$. Such a method PI proposes to develop. There were no methods for deriving an integral equation for the effective EM field in the medium in which many small impedance bodies are embedded. PI proposes to derive such an equation and give on this basis a method for creating materials with a desired refraction coefficient and/or desired magnetic permeability.

In Rayleigh theory of wave scattering by small bodies the order of the scattering amplitude is $O\left(a^{3}\right)$, where $a$ is the characteristic size of the small body, $a \rightarrow 0$. In PI's theory of wave scattering by small impedance body the scattering amplitude is of the order $O\left(a^{2-\kappa}\right)$, where $\kappa \in[0,1)$. Thus, in PI's theory the order of the scattering amplitude is much larger that in Rayleigh's theory. This difference comes from the fact that in Rayleigh's theory the scattering amplitude is proportional to the volume of the small body, while in PI's theory it is proportional to $\zeta|S|$, where $|S|=O\left(a^{2}\right)$ is the surface area of the small body, and $\zeta=O\left(a^{-\kappa}\right)$ is its boundary impedance. This difference may be used practically.

The ideas of this research were developed in [6-15]. In [16] numerical results, based on this proposal, are presented.

Remark. If Equation (7) is assumed for one small body $D$, where $v_{E}:=\nabla \times \int_{S} g(x, t) \sigma(t) d t$ and $v_{H}:=\frac{\nabla \times v_{E}}{i \omega \mu}$, then

$$
\nabla \times v_{E}=i \omega \mu v_{H} \quad i n D^{\prime}
$$

and one can derive the following equations:

$$
\begin{array}{cc}
\nabla \times v_{H}=-i \omega \epsilon v_{E} & i n D^{\prime} \\
\nabla \times \nabla \times v_{E}=k^{2} v_{E} & i n D^{\prime} \\
\nabla \times \nabla \times v_{H}=k^{2} v_{H} & i n D^{\prime}
\end{array}
$$




\section{Conflicts of Interest}

The author declares no conflict of interest.

\section{References}

1. Balay, S.; Brown, J.; Buschelman, K.; Gropp, W.; Kaushik, D.; Knepley, M.; McInnes, L.; Smith, B.; Zhang, H. PETSc Web Page. Available online: http://www.mcs.anl.gov/petsc (accessed on 24 January 2014).

2. Bohren, C.; Huffman, D. Absorption and Scattering of Light by Small Particles; Wiley: New York, NY, USA, 1998.

3. Van de Hulst, H. Light Scattering by Small Particles; Dover: New York, NY, USA, 1981.

4. Landau, L.; Lifshitz, E. Electrodynamics of Continuous Media; Pergamon Press: London, UK, 1982.

5. Mie, G. Beiträge zur Optik Trüber Medien, speziell kolloidaler Metallösungen. Ann. der Phys. 1908, 25, 377-445.

6. Ramm, A.G. Approximate formulas for polarizability tensors and capacitances of bodies of arbitrary shapes and applications. Doklady Acad. Sci. USSR 1970, 195, 1303-1306.

7. Ramm, A.G. Iterative Methods for Calculating Static Fields and Wave Scattering by Small Bodies; Springer Verlag: New York, NY, USA, 1982.

8. Ramm, A.G. Wave Scattering by Small Bodies Of Arbitrary Shapes; World Science Publishers: Singapore, 2005.

9. Ramm, A.G. Electromagnetic wave scattering by many conducting small particles. J. Phys. A 2008, 41, 212001.

10. Ramm, A.G. Electromagnetic wave scattering by small bodies. Phys. Lett. A 2008, 372, 4298-4306.

11. Ramm, A.G. Wave scattering by many small particles embedded in a medium. Phys. Lett. A 2008, 372, 3064-3070.

12. Ramm, A.G. Wave scattering by many small bodies and creating materials with a desired refraction coefficient. Afrika Matematika 2011, 22, 33-55.

13. Ramm, A.G. Electromagnetic wave scattering by many small perfectly conducting particles of an arbitrary shape. Opt. Commun. 2012, 285, 3679-3683.

14. Ramm, A.G. Scattering of electromagnetic waves by many nano-wires. Mathematics 2013, 1, 89-99.

15. Ramm, A.G. Scattering of Acoustic and Electromagnetic Waves by Small Bodies of Arbitrary Shapes. Applications to Creating New Engineered Materials; Momentum Press: New York, NY, USA, 2013.

16. Ramm, A.G.; Andriychuk, M.I. Application of the asymptotic solution to EM wave scattering problem to creating medium with a prescribed permeability. J. Appl. Math. Comput. 2013, 2013, doi:10.1007/s12190-013-0732-7.

17. Rayleigh, L. Scientific Papers; University Press: Cambridge, UK, 1922.

18. Saad, Y. ; Schulz, M. GMRES: A generalized minimal residual algorithm for solving nonsymmetric linear systems. SIAM J. Sci. Stat. Comput. 1986, 7, 856-869. 
19. Stratton, J.A. Electromagnetic Theory; Wiley: Hoboken, NJ, USA, 2007.

(c) 2014 by the author; licensee MDPI, Basel, Switzerland. This article is an open access article distributed under the terms and conditions of the Creative Commons Attribution license (http://creativecommons.org/licenses/by/3.0/). 